\title{
Effects of alveolar bone displacement with segmental osteotomy: micro-CT and histomorphometric analysis in rats
}

\author{
Taegun $\mathrm{KIM}^{(\mathrm{a})}$ \\ Won LEE(b) \\ Sang-Ho BAEK(c) \\ Sungwoon PYO(d) \\ Yoon-Ah KOOK(e) \\ Mohamed BAYOME(e) \\ Insoo $\mathrm{KIM}^{(\mathrm{b})}$ \\ (a) Catholic University of Korea, College of \\ Medicine, Seoul, Korea. \\ (b) Catholic University of Korea, College of \\ Medicine, Uijeongbu St. Mary's Hospital, \\ Department of Dentistry, Uijeongbu-si, Korea. \\ (c)Ajou University, College of Natural \\ Sciences, Department of Life Sciences, \\ Suwon, Korea. \\ (d) Catholic University of Korea, College of \\ Medicine, Bucheon St. Mary's Hospital, \\ Department of Dentistry, Seoul, Korea. \\ (e) Catholic University of Korea, College \\ of Medicine, Seoul St. Mary's Hospital, \\ Department of Dentistry, Seoul, Korea.
}

Declaration of Interests: The authors certify that they have no commercial or associative interest that represents a conflict of interest in connection with the manuscript.

\section{Corresponding Author:}

Dr. Won Lee

E-mail:cmfs21@catholic.ac.kr

DOI: 10.1590/1807-3107BOR-2016.vol30.0132

Submitted: Nov 02, 2015

Accepted for publication: Sep 23, 2016

Last revision: Oct 05, 2016
Abstract: The purpose of this study was to evaluate the effects of segmental osteotomy on the blood vessels and osteoclasts in rats using micro-computed tomography (micro-CT) and histomorphometric analysis. After segmental osteotomy was performed around the maxillary first molars of 36 male Sprague-Dawley rats $(n=72)$, the samples were divided into a control group (no displacement), $0.5 \mathrm{D}$ group $(0.5 \mathrm{~mm}$ buccal displacement) and $1.0 \mathrm{D}$ group (1.0 mm buccal displacement) ( $n=24$ /group). At 1, 2, 4 and 8 weeks after surgery, changes in the blood vessel volume were investigated using micro-CT with perfusion of radiopaque silicone rubber. Tartrate-resistant acid phosphatase (TRAP) staining was used for histomorphometric analysis. Two-way repeated measures analysis of variance (rmANOVA) was performed to compare the volume of blood vessels and number of TRAP-positive osteoclasts among the groups. Regarding blood vessel volume, the displacement groups had no significant effects, while the time points had significant effects $(p=0.014)$. The blood vessel volume at 1 week was significantly smaller than that at 2, 4, and 8 weeks $(p=0.004, p=0.026$, and $p=0.005$, respectively). Regarding TRAP cell count, the displacement groups had no significant effects, while the time points had significant effects $(\mathrm{p}<0.001)$. The number of TRAP-positive osteoclasts at 8 weeks was significantly smaller than that at 1,2 , and 4 weeks $(p<0.001, p<0.001$, and $p=0.002$, respectively), and the count at 4 weeks was smaller than that at 1 week $(p=0.011)$. Therefore, a regional osteoclast-related acceleratory phenomenon was maintained until 4 weeks after surgery.

Keywords: Alveolar Bone Displacement; Osteotomy; Blood Vessels.

\section{Introduction}

Asian orthodontic patients are known to have bimaxillary protrusion. If the amount of the anterior tooth retraction is large, there is the possibility of root resorption and/or exposure and lingual tipping of the anterior teeth after orthodontic treatment. Therefore, it is necessary to perform a surgical approach that can move the teeth and bone at the same time., ${ }^{1,2}$ One of the methods, alveolar segmental osteotomy, attempts to shorten the duration of orthodontic treatment and to minimize the side effects by orthodontic treatment. ${ }^{3,4,5}$

The amount of surgical movement of the osteomized segment is important because it can affect blood circulation. ${ }^{6}$ Therefore, surgeons 
should prevent the occurrence of ischemia in the osteomized segment and should keep it within a range with no effect on the blood circulation. ${ }^{78}$ In addition, this surgical method also has the possibility of complications, such as necrosis of the gingival and periodontal tissue, abnormal sensations in the teeth due to dental pulp degeneration, and direct damage to the tooth root. ${ }^{9}$ If the changes in the soft and hard tissues after segmental osteotomy can be determined, it might increase understanding of the effects of segmental osteotomy on the teeth, alveolar bone, and periodontal tissue, thus minimizing complications.

Although there have been several previous studies that investigated blood circulation using two-dimensional (2D) micro-angiography and histological observation after segmental osteotomy, ${ }^{7,8,10}$ three-dimensional (3D) study using micro-computed tomography (CT) has not been performed. Therefore, the purpose of this study was to evaluate the effects of alveolar bone displacement with segmental osteotomy on the blood vessels and osteoclasts in the area of the maxillary first molar in rats using micro-CT and histomorphometric analysis.

\section{Methodology}

\section{Experimental animals}

The sample consisted of 36 male Sprague-Dawley rats (body weight range, 296-345 g). The approval of study was obtained from the Institutional Review Board of the Catholic University of Korea, School of Medicine (approval no. UJA2012-19A). The study was conducted at the clinical study center of Bucheon St. Mary's Hospital, College of Medicine, the Catholic University of Korea. All of the analyses were performed by one investigator. The sample size was calculated using GLIMMPSE software, version 2.2.1 (University of Colorado Denver, Denver, Colorado, USA) and revealed that 24 samples were required for each group.

\section{Surgical technique}

A mixture of $50 \mathrm{mg} / \mathrm{kg}$ ketamine (Yuhan Ketamin 50 inj, Yuhan Corporation, Seoul, Korea) and $10 \mathrm{mg} / \mathrm{kg}$ Rumpun (Rumpun 2\% inj, Bayer Korea Seoul, Seoul, Korea) was injected intraperitoneally to induce general anesthesia. Segmental osteotomy was performed around the right and left maxillary first molars of the 36 rats $(\mathrm{n}=72)$. Then, using a split-mouth design, the sample was divided into three equal groups according to the amounts of buccal displacement of the alveolar bone segment [control group (no displacement), $0.5 \mathrm{D}$ group $(0.5 \mathrm{~mm}$ displacement) and 1.0 D group (1.0 mm displacement)] ( $n=24$ per group). The osteomized segment was fixed with the adjacent teeth by glass ionomer cement (GI) after suture, and the opposing teeth in the mandible were removed for the prevention of occlusal contact (Figure 1).

\section{Animal sacrifice}

The animals were sacrificed at one, two, four, and eight weeks after the procedure (9 each week). After general anesthesia, cleaning with heparinized physiological saline and perfusion fixation using $4 \%$ paraformaldehyde were performed.

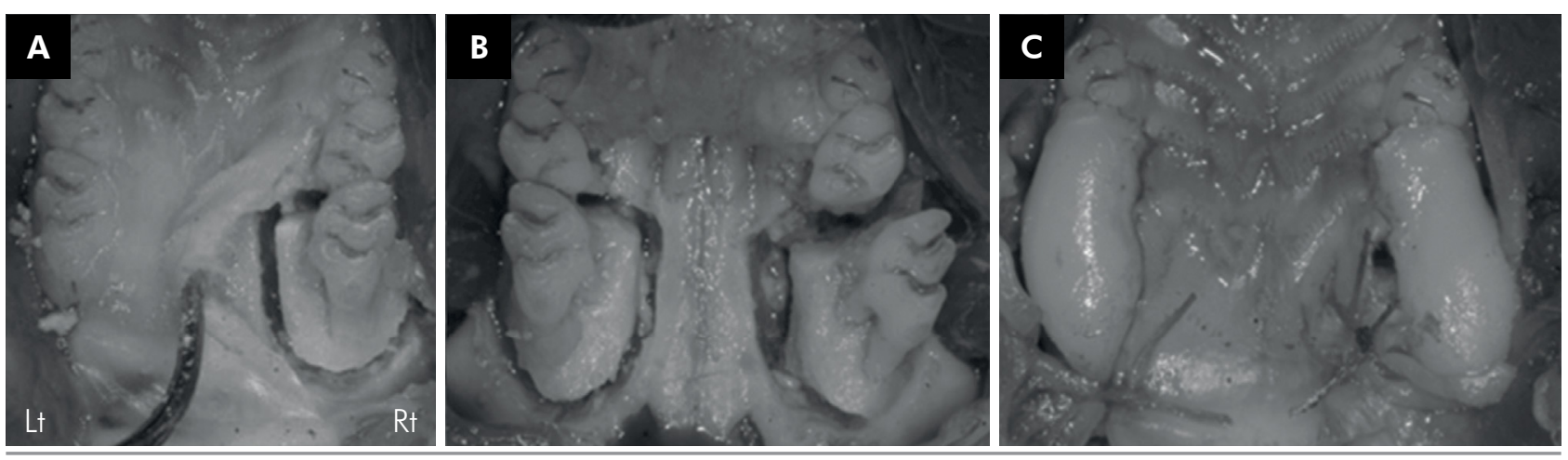

Figure 1. Design of the alveolar segmental osteotomy. A) After a mucoperiosteal flap was elevated on the first maxillary molar, the alveolar segmental osteotomy was performed. B) The bony segment of the experimental group was buccally displaced (right), while the control group was not displaced (left). C) After the flap was sutured, the bony segments were fixed with a light-curing glass ionomer (GI) cement. 


\section{D micro-CT analysis}

To observe changes in the blood vessels, radiopaque silicon (Microfil ${ }^{\circledR}$, MV122, FlowTech, Carver, USA) was injected. After the silicon hardened, the maxilla, including experiment site, was separated and fixed in $4 \%$ paraformaldehyde for one week. Then, the maxilla was washed with running water for 2-3 days and was decalcified by a mixture of 15\% EDTA (ethylene diamine tetra-acetic acid) and $4 \%$ sucrose in a microwave issue processor (Pelco BioWave Pro, Ted Pella Inc., Redding, USA) for three weeks.

Micro-CT (SkyScan1172, Bruker microCT, Kontich, Belgium) was performed at the level of $12 \mu \mathrm{m}$ voxel size. The blood vessel volume at the site of segmental osteotomy was analyzed at a volume of $3.5 \times 4.5 \times 2.5 \mathrm{~mm}^{3}$ using CTAn software (version 1.16) (Bruker microCT, Kontich, Belgium).

\section{Tissue preparation}

The samples were decalcified by a mixture of $15 \%$ EDTA and $4 \%$ sucrose in a microwave tissue processor (Ted Pella Inc.) for additional five weeks after micro-CT examination. The decalcified tissues were embedded in paraffin with vacuum infiltration tissue processors (VIP 5J-F2, Sacura Finetek Japan, Tokyo, Japan) after cleaning them for 2-3 days. They were cut at thicknesses of $4 \mu \mathrm{m}$ parallel to the occlusal plane using a microtome (RM2255, Leica, Nussloch, Germany). After cutting $1 / 3$ of the maxillary first molar root, transverse sections were dyed with hematoxylin and eosin (H\&E) and were observed under an optical microscope (BX 53F/DP72, Olympus, Tokyo, Japan).

\section{Staining for Tartrate-resistant acid phosphatase (TRAP)}

For the staining of osteoclasts, a TRAP and ALP double stain kit (\#MK300, Takara, Shiga, Japan) was used. After sectioning of the paraffin-embedded tissue at $4 \mu \mathrm{m}$ of thickness, a deparaffinization process using xylene was repeated three times for five minutes each. After being submitted to a hydration process with $100 \%, 90 \%, 70 \%$, and $50 \%$ ethanol for one minute each, the sections were cleaned with distilled water. An ACP staining solution was undertaken at $37^{\circ} \mathrm{C}$ for one hour. Again, methyl green staining was performed for ten minutes after cleaning with distilled water. After soaking in distilled water ten times, they were mounted to determine the number of osteoclasts with an optical microscope. The TRAPpositive cells were counted in a square of $1 \times 1 \mathrm{~mm}^{2}$ at the furcation area of the first molar root.

\section{Statistical analysis}

Statistical analysis was performed using IBM SPSS statistics software, version 20 (IBM Co., New York, USA). The comparisons between groups and time points regarding the volumes of blood vessels and numbers of TRAP-positive osteoclasts were performed using two-way repeated measures analysis of variance (rmANOVA) with Bonferroni's post hoc lest for multiple comparisons. The significance level was set at $\mathrm{p}<0.05$.

\section{Results}

\section{Change in the blood vessels}

Although there was no significant main effect of the displacement groups (control, $0.5 \mathrm{D}$ and $1.0 \mathrm{D}$ groups; $p=0.974$, Table 1$)$, there were significant main effects of the time points $(p=0.014$, Table 1$)$. Additionally, there were no significant interactions between displacement and time points $(\mathrm{p}=0.774$, Table 1). The multiple comparisons between the time points demonstrated that the volume of the blood vessels at 1 week was significantly smaller than the volumes at 2,4 , and 8 weeks ( $p=0.004, p=0.026$, and $\mathrm{p}=0.005$, respectively; Table 1 and Figures 2 and 3)

\section{Osteoclast measurement}

Osteoclast measurement showed similar results to blood vessel volume (Table 2 and Figures 4 and 5). Despite no significant main effects of the displacement groups (control, 0.5 D and 1.0 D groups; $p=0.351$, Table 2), there was a significant main effect of the time points $(\mathrm{p}<0.001$, Table 2$)$. Additionally, there were no significant interactions among the time points ( $p=0.172$, Table 2 ). The multiple comparisons between the time points demonstrated that the number of TRAP-positive osteoclasts at 8 weeks was significantly smaller than at 1,2, and 4 weeks $(\mathrm{p}<0.001, \mathrm{p}<0.001$, and $\mathrm{p}=0.002$, respectively; 
Table 2), and the count at 4 weeks was also smaller than at 1 week $(p=0.011$, Table 2$)$.

\section{Discussion}

In alveolar segmental osteotomy, the determination of the range of stable displacement of the segment can reduce complications such as necrosis, which occurs if blood flow to the segment is not maintained. ${ }^{5}$ In previous studies using micro-angiography in monkeys, blood supply within the bone marrow was discontinued during segmental osteotomy, and recovery of the blood supply occurred through a number of capillaries in the periodontal tissue. ${ }^{10,11}$ Although there have been numerous studies using laser Doppler, spectrophotometry and flow cytometry, they focused on identifying the anatomical structures of the blood vessel and the overall blood flow rate. ${ }^{12,13,14,15}$
Recently, more accurate analyses of blood flow, using a silicone rubber compound and micro-CT, have been conducted..$^{16,17}$ Donneys et al. ${ }^{18,19}$ analyzed the vascular changes after mandibular osteotomy in rats, and they observed a significant increase in the blood vessel volume during osteogenesis. However, there have been few 3D analysis studies of the vascular volume after segmental osteotomy. Micro-CT enabled the detection of minimal changes in the bones and capillaries (Figure 2). However, both high definition and a low threshold level are required to increase the accuracy of micro-CT studies. ${ }^{17}$ Therefore, in our study, to obtain the 3D images, the definition was set at $12 \mu \mathrm{m}$, and the threshold was set at 60 .

In terms of the change in blood vessel volume, the vascular volume at 1 week after surgery was significantly smaller than at 2, 4, and 8 weeks after surgery $(p=0.004, p=0.026$, and $p=0.005$,
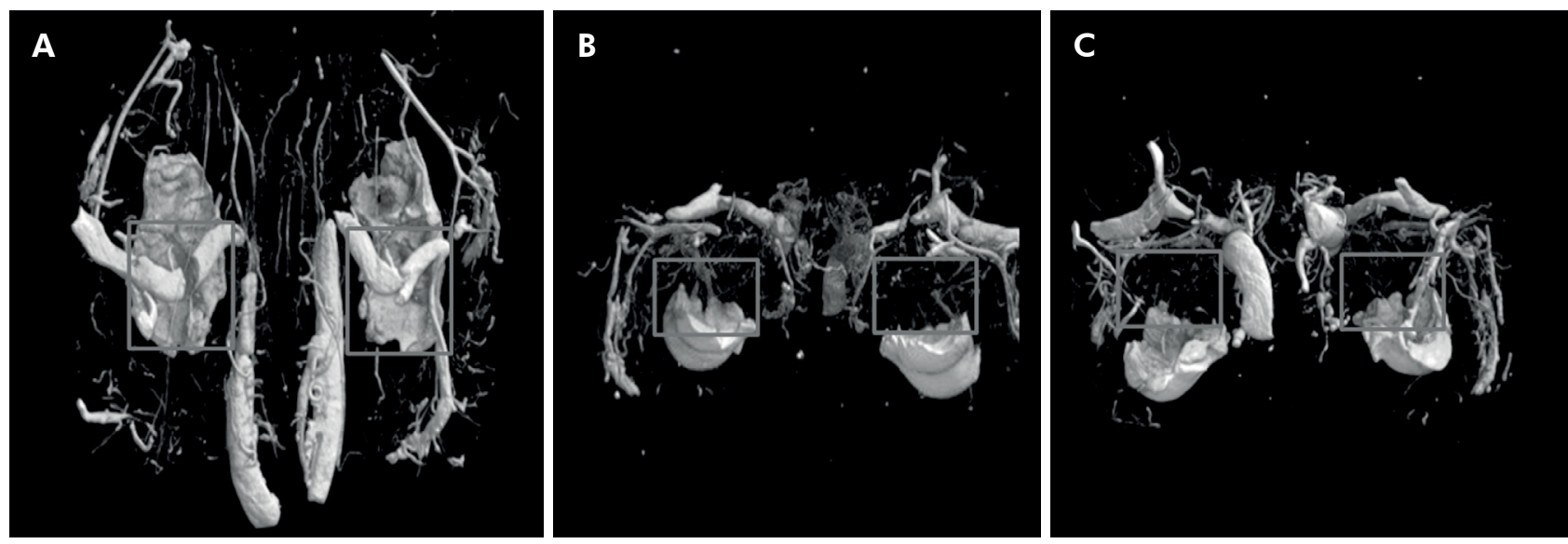

Figure 2. The occlusal (A), frontal (B) and rear (C) views of the blood vessels in the maxilla at 1 week after surgery $(0.5 \mathrm{~mm}$ and $1.0 \mathrm{~mm}$ displacement groups). Each image within the solid line box $\left(3.5 \times 4.5 \times 2.5 \mathrm{~mm}^{3}\right.$, horizontal, vertical, height measurements, respectively) was analyzed. The $\mathrm{Gl}$ cement appeared radiopaque.

Table 1. Comparison of blood vessel volume according to time point and group.

\begin{tabular}{|c|c|c|c|c|c|c|c|c|c|c|c|}
\hline \multirow{3}{*}{$\begin{array}{l}\text { Time point } \\
\text { Group } \\
0.5 \mathrm{~mm} \text { deviation group }\end{array}$} & \multicolumn{2}{|c|}{1 week } & \multicolumn{2}{|c|}{2 weeks } & \multicolumn{2}{|c|}{4 weeks } & \multicolumn{2}{|c|}{8 weeks } & \multirow{2}{*}{$\begin{array}{c}p \text {-value } \\
\text { (time points) }\end{array}$} & \multirow{2}{*}{\multicolumn{2}{|c|}{$\begin{array}{l}\text { Multiple comparison according } \\
\text { to time point }\end{array}$}} \\
\hline & Mean & SD & Mean & SD & Mean & SD & Mean & SD & & & \\
\hline & 1.20 & 1.01 & 2.07 & 1.19 & 2.02 & 0.65 & 1.79 & 0.90 & \multirow{3}{*}{0.014} & $1 W<2 W$ & $p=0.004$ \\
\hline $1.0 \mathrm{~mm}$ deviation group & 0.62 & 0.31 & 2.18 & 0.70 & 1.64 & 0.09 & 2.52 & 0.19 & & $1 W<4 W$ & $p=0.026$ \\
\hline Control & 0.29 & 0.12 & 2.64 & 0.37 & 1.91 & 0.53 & 2.48 & 1.66 & & $1 W<8 W$ & $p=0.005$ \\
\hline $\mathrm{p}$-value (displacement) & & & & & & & & & $\begin{array}{l}\text { Interaction: } \\
\mathrm{p}=0.774\end{array}$ & & \\
\hline
\end{tabular}

Two-way repeated measures ANOVA with Bonferroni's post hoc test 


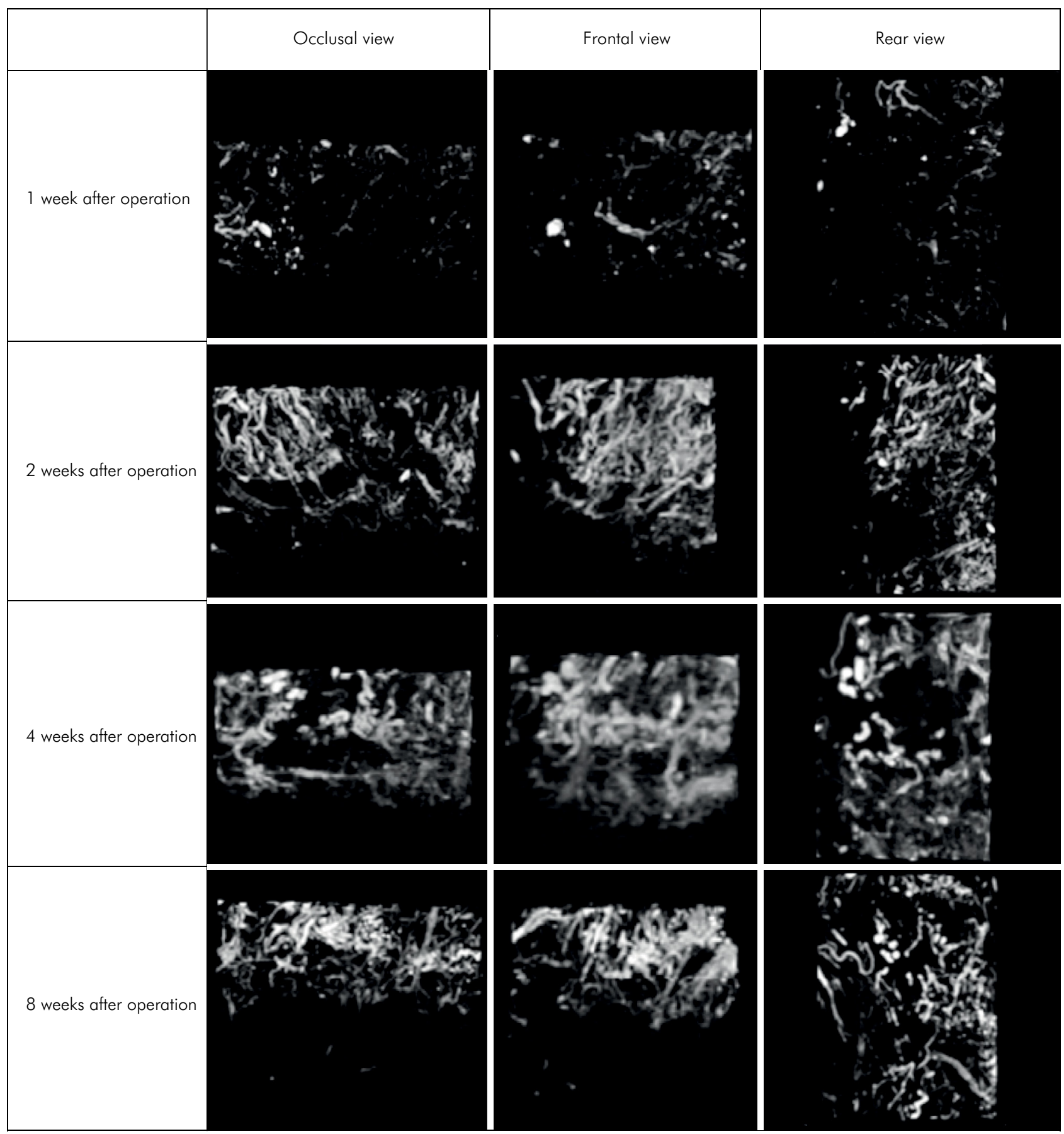

Figure 3. Distribution of the blood vessels adjacent to the osteotomy site at 1, 2, 4 and 8 weeks after surgery (1.0 mm displacement group).

respectively; Table 1). Zhao et al. ${ }^{20}$ reported that the blood flow would increase if fixation were performed securely after osteotomy. Therefore, in our study, the maxillary first molar in the segment was fixed to the adjacent teeth using light-curing GI cement, and the opposing tooth in the mandible was removed to prevent interference with the segment stability (Figure 1). Therefore, in segmental osteotomy, early stabilization of the displaced segment might be important for maintaining initial blood circulation.

Donneys et al. ${ }^{19}$ and Wang et al. ${ }^{21}$ reported that there was no significant difference in the numbers of 

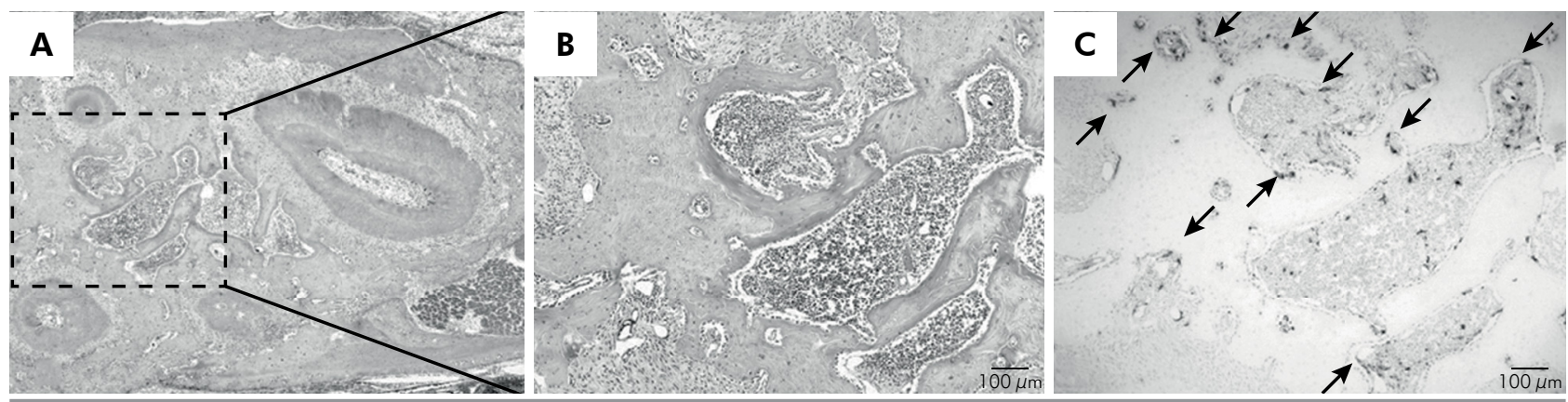

Figure 4. TRAP-positive osteoclasts in the $0.5 \mathrm{~mm}$ displacement group at 1 week after segmental osteotomy on the maxillary first molar. A: T.S. at the root region of the maxillary first molar. B: Magnified view of the dotted box in A $\left(1 \times 1 \mathrm{~mm}^{2} \mathrm{area}_{\text {between the }}\right.$ roots of the maxillary first molar). C: TRAP-positive cells were counted within this dotted box.

Table 2. Comparison of TRAP cell count according to time point and group.

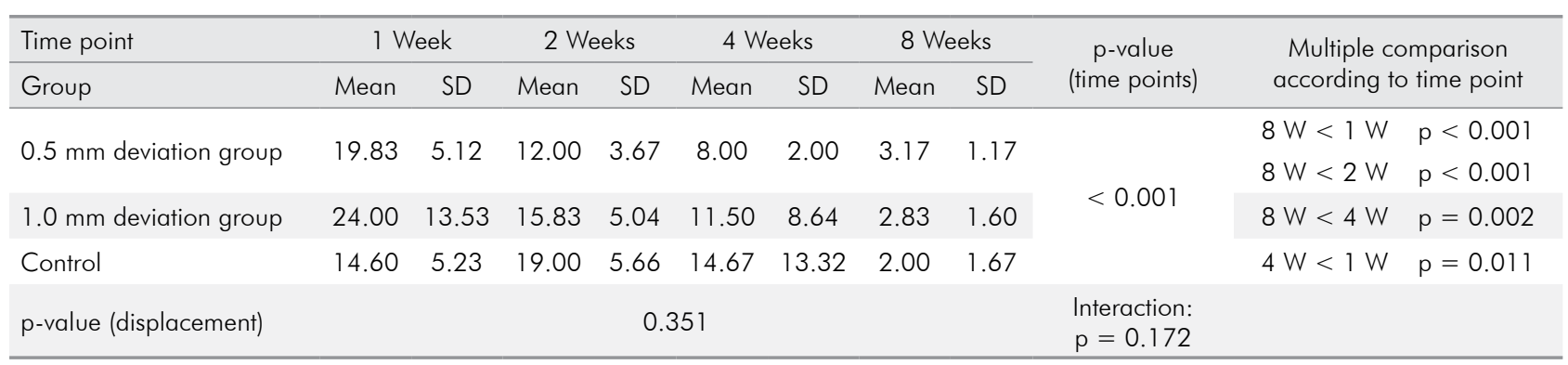

Two-way repeated measures ANOVA with Bonferroni's post hoc test.

vessels between osteomized bone and an untreated control group using micro-angiography. However, Claes et al. ${ }^{22}$ observed that, as the distance between the segment and basal bone increased, the soft tissue with fibrous cartilage increased, and the number of new blood vessels decreased. However, in our results, there was no significant difference in the vascular volume according to the amount of segment displacement (Table 1). Therefore, $1.0 \mathrm{~mm}$ displacement of the segment in rats might not significantly affect the production of new blood vessels.

Under normal circumstances, tartrate-resistant acid phosphatase enzyme is highly expressed by osteoclasts; therefore, TRAP staining was applied to evaluate the presence and activity of osteoclasts in the specimens. The presence of active osteoclasts is associated with bone remodeling which in turn is linked to tooth movement. In our study, the number of TRAP-positive osteoclasts was maintained until 4 weeks after surgery, and it decreased at 8 weeks after segmental osteotomy ( 1 week vs. 8 weeks, $p<0.001 ; 2$ weeks vs. 8 weeks, $\mathrm{p}<0.001$; and 4 weeks vs. 8 weeks, $p=0.002$, respectively; Table 2), indicating that there was interaction between the healing period and osteoclast activity. However, Wang et al. ${ }^{21}$ and Sebaoun et al. ${ }^{23}$ reported that the number of osteoclasts was greatest at 2 and 3 weeks after corticotomy in white rats, and it tended to decrease significantly from 4 weeks after corticotomy. In our study, however, the number of osteoclasts significantly decreased at 8 weeks after osteotomy, leading to the inference that osteoclast activity became longer in osteotomy than in corticotomy.

In our study, in all cases, regardless of the displacement amount, the vascular volume was smallest at 1 week after segmental osteotomy (Table 1). Since maintenance of the initial blood circulation after surgical procedures is considered important for healing of the segment, the segment should have been fixed to obtain sufficient and stable contact with the adjacent bones and to avoid the possibility of failure of bony fusion. . $2,24,25^{2}$ Early stabilization of the displaced segment might be important for maintaining initial blood circulation. 


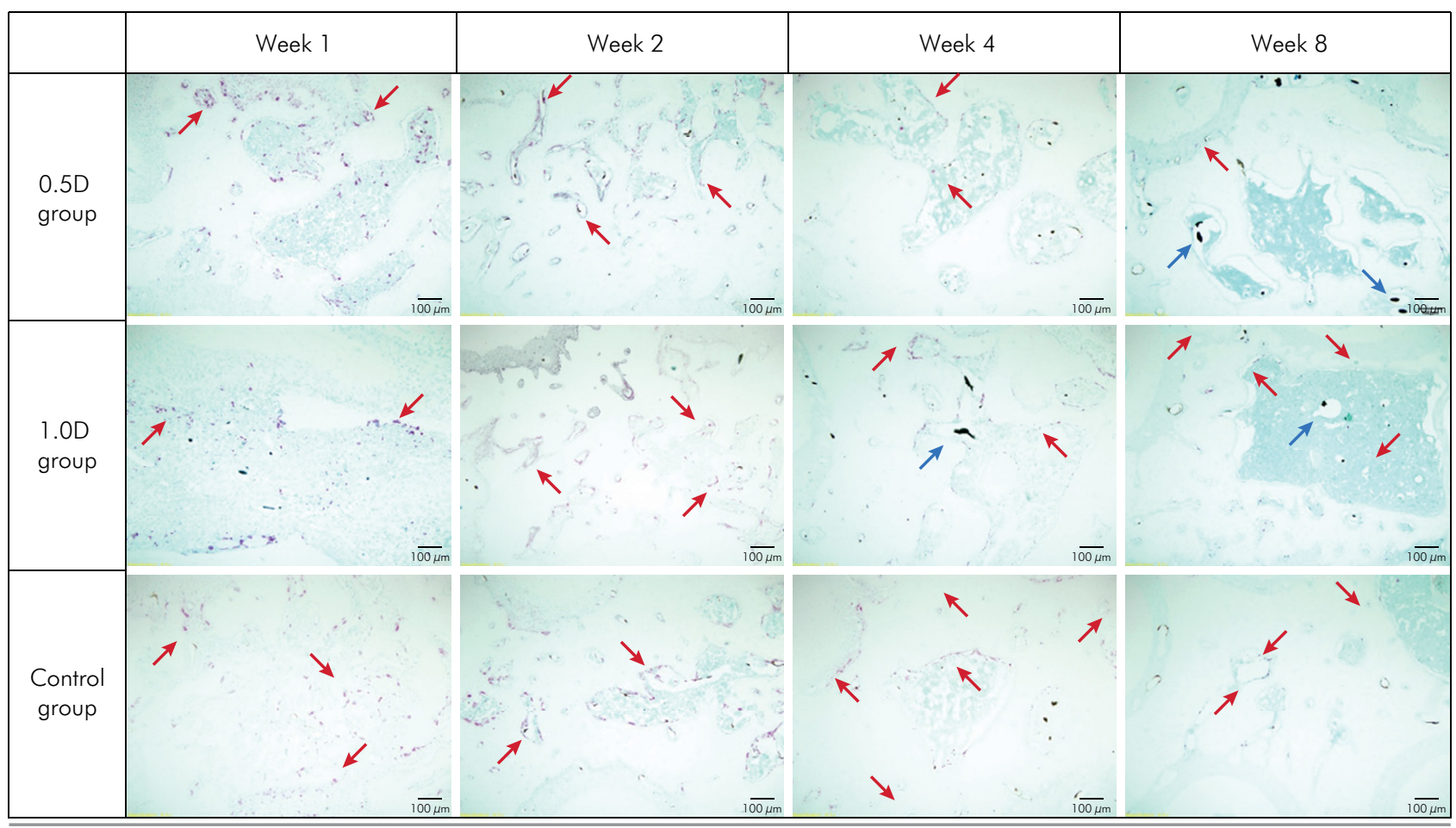

Figure 5. Changes in the TRAP-positive osteoclasts according to the time period after segmental osteotomy ( $1.0 \mathrm{~mm}$ displacement group). Red arrows, TRAP-positive osteoclasts; blue arrows, radiopaque silicone rubber in the blood vessels.

Osteoclast activity remained high up to 4 weeks after segmental osteotomy (Table 2), suggesting that the regional acceleratory phenomenon (RAP) was continued until that time. Hence, the speed of orthodontic tooth movement in white rats might be faster from 1 to 4 weeks after surgery than at 8 weeks after surgery. Interestingly, because white rats are known to have three times more rapid bone metabolism than humans, post-operative orthodontic treatment in humans would be optimally conducted for 3 to 12 weeks after surgery.

\section{References}

1. Chung KR, Oh MY, Ko SJ. Corticotomy-assisted orthodontics. J Clin Orthod. 2001;35(5):331-9.

2. Köle H. Surgical operations on the alveolar ridge to correct occlusal abnormalities. Oral Surg Oral Med Oral Pathol. 1959;12(3):277-88. doi: 10.1016/0030-4220(59)90177-X

3. Chung KR, Kim SH, Lee BS. Speedy surgical-orthodontic treatment with temporary anchorage devices as an alternative to orthognathic surgery. Am J Orthod Dentofacial Orthop. 2009;135(6):787-98. doi:10.1016/j.ajodo.2007.03.036

4. Jacobs JD, Bell WH. Combined surgical and orthodontic treatment of bimaxillary protrusion. Am J Orthod. 1983;83(4):321-33. doi:10.1016/0002-9416(83)90228-2

\section{Conclusions}

The blood vessel volume at 1 week was significantly smaller than that at 2, 4, and 8 weeks. However, there was no significant effect of the displacement of the segment on the volume of the blood vessel.

TRAP analysis showed that osteoclast-related RAP lasted 4 weeks after surgical procedures in rats. It can be suggested that osteoclast-related RAP might be maintained for 4 weeks after segmental osteotomy.

5. Lanigan DT, Hey JH, West RA. Aseptic necrosis following maxillary osteotomies: report of 36 cases. J Oral Maxillofac Surg. 1990;48(2):142-56. doi:10.1016/S0278-2391(10)80202-2

6. Chung KR, Mitsugi M, Lee BS, Kanno T, Lee W, Kim $\mathrm{SH}$. Speedy surgical orthodontic treatment with skeletal anchorage in adults: sagittal correction and open bite correction. J Oral Maxillofac Surg. 2009;67(10):2130-48. doi:10.1016/j.joms.2009.07.002

7. Bell WH, Levy BM. Revascularization and bone healing after anterior mandibular osteotomy. J Oral Surg. 1970;28(3):196-203. 
8. Bell WH, You ZH, Finn RA, Fields RT. Wound healing after multisegmental Le Fort I osteotomy and transection of the descending palatine vessels. J Oral Maxillofac Surg. 1995;53(12):1425-33. doi:10.1016/0278-2391(95)90670-3

9. Parnes EI, Becker ML. Necrosis of the anterior maxilla following osteotomy. Oral Surg Oral Med Oral Pathol. 1972;33(3):326-30. doi:10.1016/0030-4220(72)90460-4

10. Bell WH. Revascularization and bone healing after anterior maxillary osteotomy: a study using adult rhesus monkeys. J Oral Surg. 1969;27(4):249-55.

11. Bell WH, Schendel SA, Finn RA. Revascularization after surgical repositioning of one-tooth dento-osseous segments. J Oral Surg. 1978;36(10):757-65.

12. Brady JM, Cutright DE. A new technique of measuring blood vessel volume in bone applied to the mandible and humerus of the rat. Anat Rec. 1971;170(2):143-6. doi:10.1002/ar.1091700203

13. Couffinhal T, Silver M, Zheng LP, Kearney M, Witzenbichler B, Isner JM. Mouse model of angiogenesis. Am J Pathol. 1998;152(6):1667-79.

14. Cutright DE. The proliferation of blood vessels in gingival wounds. J Periodontol. 1969;40(3):137-41. doi:10.1902/jop.1969.40.3.137

15. Cutright DE, Bhaskar SN. Pulpal vasculature as demonstrated by a new method. Oral Surg Oral Med Oral Pathol. 1969;27(5):678-83. doi:10.1016/0030-4220(69)90104-2

16. Bentley MD, Ortiz MC, Ritman EL, Romero JC. The use of microcomputed tomography to study microvasculature in small rodents. Am J Physiol Regul Integr Comp Physiol. 2002;282(5):R1267-79. doi:10.1152/ajpregu.00560.2001

17. Duvall CL, Taylor WR, Weiss D, Guldberg RE. Quantitative microcomputed tomography analysis of collateral vessel development after ischemic injury. Am J Physiol Heart Circ Physiol. 2004;287(1):H302-10. doi:10.1152/ajpheart.00928.2003
18. Donneys A, Tchanque-Fossuo CN, Farberg AS, DeshpandeSS, Buchman SR. Bone regeneration in distraction osteogenesis demonstrates significantly increased vascularity in comparison to fracture repair in the mandible. J Craniofac Surg. 2012;23(1):328-32. doi:10.1097/SCS.0b013e318241db26

19. Donneys A, Tchanque-Fossuo CN, Farberg AS, Jing XL, Deshpande SS, Goldstein SA, et al. Quantitative analysis of vascular response after mandibular fracture repair using microcomputed tomography with vessel perfusion. Plast Reconstr Surg. 2011;127(4):1487-93. doi:10.1097/PRS.0b013e318208f3c1

20. Zhao F, Zhou Z, Yan Y, Yuan Z, Yang G, Yu H et al. Effect of fixation on neovascularization during bone healing. Med Eng Phys. 2014;36(11):1436-42. doi:10.1016/j.medengphy.2014.07.002

21. Wang L, Lee W, Lei DL, Liu YP, Yamashita DD, Yen SL. Tisssue responses in corticotomy- and osteotomy-assisted tooth movements in rats: histology and immunostaining. Am J Orthod Dentofacial Orthop. 2009;136(6):770 e1-11. doi:10.1016/j.ajodo.2009.05.015.

22. Claes L, Eckert-Hubner K, Augat P. The fracture gap size influences the local vascularization and tissue differentiation in callus healing. Langenbecks Arch Surg. 2003;388(5):316-22. doi:10.1007/s00423-003-0396-0

23. Sebaoun JD, Kantarci A, Turner JW, Carvalho RS, Van Dyke TE, Ferguson DJ. Modeling of trabecular bone and lamina dura following selective alveolar decortication in rats. J Periodontol. 2008;79(9):1679-88. doi:10.1902/jop.2008.080024

24. Augat P, Margevicius K, Simon J, Wolf S, Suger G, Claes L. Local tissue properties in bone healing: influence of size and stability of the osteotomy gap. J Orthop Res. 1998;16(4):475-81. doi:10.1002/jor.1100160413

25. Markel MD, Bogdanske JJ. The effect of increasing gap width on localized densitometric changes within tibial ostectomies in a canine model. Calcif Tissue Int. 1994;54(2):155-9. doi:10.1007/BF00296067 